\title{
Machine Learning Assisted Cervical Cancer Detection
}

\author{
Mavra Mehmood ${ }^{1}$, Muhammad Rizwan ${ }^{1}$, Michal Gregus $\mathrm{ml}^{2 *}$ and Sidra Abbas ${ }^{3 *}$ \\ ${ }^{1}$ Department of Computer Science, Kinnaird College for Women, Lahore, Pakistan, ${ }^{2}$ Information Systems Department, \\ Faculty of Management, Comenius University in Bratislava, Bratislava, Slovakia, ${ }^{3}$ ASETS Lab, Islamabad, Pakistan
}

\section{OPEN ACCESS}

Edited by:

Thippa Reddy Gadekallu, VIT University, India

Reviewed by: Weizheng Wang

City University of Hong Kong, Hong

Kong SAR, China

Giridhar Boija

Dakota State University, United States

*Correspondence:

Sidra Abbas

sidra.abbas708@gmail.com Michal Gregus ml michal.gregus.m/@fm.uniba.sk

Specialty section: This article was submitted to Digital Public Health,

a section of the journal

Frontiers in Public Health

Received: 02 October 2021 Accepted: 16 November 2021

Published: 23 December 2021

Citation:

Mehmood M, Rizwan M, Gregus m/ M and Abbas S (2021) Machine Learning

Assisted Cervical Cancer Detection.

Front. Public Health 9:788376.

doi: 10.3389/fpubh.2021.788376
Cervical malignant growth is the fourth most typical reason for disease demise in women around the globe. Cervical cancer growth is related to human papillomavirus (HPV) contamination. Early screening made cervical cancer a preventable disease that results in minimizing the global burden of cervical cancer. In developing countries, women do not approach sufficient screening programs because of the costly procedures to undergo examination regularly, scarce awareness, and lack of access to the medical center. In this manner, the expectation of the individual patient's risk becomes very high. There are many risk factors relevant to malignant cervical formation. This paper proposes an approach named CervDetect that uses machine learning algorithms to evaluate the risk elements of malignant cervical formation. CervDetect uses Pearson correlation between input variables as well as with the output variable to pre-process the data. CervDetect uses the random forest (RF) feature selection technique to select significant features. Finally, CervDetect uses a hybrid approach by combining RF and shallow neural networks to detect Cervical Cancer. Results show that CervDetect accurately predicts cervical cancer, outperforms the state-of-the-art studies, and achieved an accuracy of 93.6\%, mean squared error (MSE) error of 0.07111 , false-positive rate (FPR) of $6.4 \%$, and false-negative rate (FNR) of 100\%.

Keywords: cervical cancer, medical data, gynecological diseases, artificial intelligence, feature engineering, classification

\section{INTRODUCTION}

Machine learning (ML) and deep learning (DL) are being utilized for brain tumor detection, cervical cancer detection, breast cancer detection, COVID detection, physical activity recognition, thermal sensation detection, and cognitive health assessment of dementia individuals $(1-3,3-$ 10). Advancements in Health Care Industry makes it more effective than traditional diagnosing techniques (11-14). According to medical reports published by Global cancer statistics (15) every year, 493,000 cervical malignancy patients have been added, among which $15 \%$ are female malignancy patients. This disease is mainly found in developing countries (16) with an $83 \%$ death ratio. Prominent in African countries (17) e.g., Uganda, which ranks fourteenth among the highest incidence of cervical malignancies with $65 \%$ of confirmed cases (18).

Human papillomavirus (HPV) contamination is mainly caused by cervical malignancy. HPV contamination is sexually transmitted. Sexual behavior associated with age at first sexual contact and the sexual activity of the accomplice is linked to the increased risk of HPV acquisition. Throughout this case, the cervical malignancy is profoundly preventable through accessible screening and detection than other different sorts of malignancy, and it is critical to actualizing 
risk expectations. The malignant cervical formation is a malignant tumor. Cervical tissue cells expand and replicate abnormally without regulated cell division and death cause. If the tumor becomes malignant, the cell travels to other areas of the body, such that certain sections become subsequently infected and, in most serious situations, may be avoided through early identification (19). Deaths due to cervical malignancies can be reduced if effective screening strategies are implemented (20). With the fast advancement of presentday clinical innovation and computer technological innovation, various screening and diagnostic strategies depend on computeraided (CAD) architectures.

A procedure of retrieving applicable information from information sources is known as data mining $(21,22)$. Realworld information comprises grimy information, for example, inaccurate and incomplete. Along these lines, cleaning and changing crude information to permit a reliable analytic delivery can accurately reflect the outcome $(23,24)$. It is implemented on the dataset. The cervical cancer dataset acquired for examination contains redundancy, missing values, and noise. Mining methods are considered one of the greatest challenges and significant fields of study in medicine due to the growing importance of health issues (25). The data mining framework can advance the cervical cancer screening process with the help of the knowledge it extracts (26). Across the medical sector, these techniques are helpful not just in finding similarities and correlations between such symptoms but also in predicting diseases. By applying several data mining techniques, continuous research and medical treatment can be immediately recommended, resulting in lifesaving, especially for cervical cancer. The first step is preprocessing $80 \%$ of information, which plays a vital role as a round of all data mining operations (27).

The random forest (RF) is used to identify essential features in our research that improve the training dataset's quality. RF constructs a forest of tree classifications in which each tree grows on a bootstrap sample data, and the characteristics of every other tree node are selected from a random subset of all characteristics. The final level of the entity is measured by voting across all trees in the forest. Several significant benefits to an RF method makes it the perfect methodology for studying particular biological data in pharmacogenomics research findings. Next, it can accommodate a wide range of both qualitative and quantitative input vectors. Second, it tests the attribute's importance in estimating the type, thereby providing a benchmark for selecting features. Third, RF generates an accurate classifier for unbiased internal generalized analysis during the forest growth process. Finally, RF is relatively stable in the face of etiological variability, and a reasonably low amount of missed data (28).

This article makes the following contributions:

- CervDetect implements ML algorithms on the medical data to gain a deeper understanding and to evaluate the risk elements of malignant cervical formation.

- Utilizes Pearson correlation between input variables and the output variable to pre-process the data.

- CervDetect uses the RF feature selection technique to select significant features.
- Proposes a Hybrid approach by combining RF and shallow neural network to detect cervical cancer.

- Effectively enhance the detection rate in comparison with state-of-the-art studies.

The rest of the part is arranged as follows. Section 2 presents the literature review. Section 3 presents the dataset and preliminaries. Section 4 presents the proposed methodology. Section 5 examines the result and discussion. Finally, section 6 presents the conclusion and future work.

\section{LITERATURE REVIEW}

In earlier studies, various methodologies based on traditional ML approaches, including k-nearest neighbors (KNN) and K-means clustering and RF, have been utilized for cervical cancer diagnosis (29-31). In 2017, WEN WU1 and HAO developed a clinical decision support network for malignant formation, using a knowledge-based method in conjunction with a rough collection of hypotheses hereditary genetic algorithms calculations in a soft computing model (32). Parallelism, selflearning, and sensitivity to internal failure are artificial neural network (ANN) characteristics. The suggested paradigm takes advantage of these characteristics. Although the information processing capabilities of the rough set theory, and the robust, parallel, and vigorous search are characteristics of the GA's. It still faces high computation problems.

Ashok, Dr. P. Aruna (33) discussed various methods of $\mathrm{ML}$, including support vector machines (SVM), gray level concurrence matrix (GLCM), KNN, convolutional neural networks (CNN), spatial fuzzy cluster algorithms, RF, C5.0, and hierarchical cluster algorithm for feature extraction, segmentation, cell classification, and evaluated in typical parameters, such as dataset volume, disadvantages, and precision. Although it is only best suited for small datasets. Chih-Jen Tseng and Chi-Jie Lu (34) have used image processing, data extraction, and ML techniques to diagnose cervical cancer. The combination of texture and shape features is extracted from each image. Optimal properties are chosen to select reciprocal information, sequential forward selection, and random section selection. Images of cervical cancer are classified using the SVM. Various selection methods are contrasted to determine the proper mechanism suitable for the diagnosis of cervical cancer. The problem faced by SVM is that there is no probabilistic explanation for classification, which leads to very rigid classifications.

In 2006, Reif et al. (35) described RF efficacy in various model genetic and proteomic datasets. RF progress fails to classify related traits based on genetic data and proteomics datasets. Experimental findings indicate that using several data sources is useful where the disease definition is uncertain, and the corresponding data basis for the phenotypic outcome is unknown. This study's findings indicate that RF is exceptional for detecting high-dimensional data vector characteristics with minimal main effects and low heritability, but the problem faced by RF chooses only one attribute at each tree split during construction, strictly epistatic. In 2016, Vidya and Nasira (36) worked on predicting the normal cervix. The cancer cervix is 
evaluated using practical data mining algorithms. Geetha and Thangamani (37) addressed the imbalanced distribution of data and risk factors for cervical cancer diagnosis.

Anuraga et al. (38) attempted to explore the conditions that affect cancer patients' survival in Makassar, Indonesia. The specimens included in this research contained as many as 38 cancer sufferers. They use the RF to identify tree merger data by combining sample data training. The main problem faced by this technique is that it only achieves $50 \%$ of accuracy. The Bandyopadhyay and Nasipuri (39) focused on K-Means clustering to segmentation pre-processed images, and Herlev analysis is carried out. IOU segmentation outcomes, verified ground truth values, and shape attributes are extracted from the segmented nucleus. The identification of the nucleus on the grounds of the shape features is carried out with the RF Classifier's aid, and the contrast is rendered with the other classifiers.

In 2020, Alyafeai and Ghouti (40) build a completely integrated cervical cancer identification and cervical cancer screening pipeline from cervical images. The current pipeline comprises two deep neural network-learning models for automated cervical identification and diagnosis of cervical tumors. The first test detects the cervix area 1,000 times faster than the state-of-the-art data-driven simulations, thus obtaining a detection precision of 0.68 in terms of union intersection (IoU) estimation. Self-extracted characteristics are used in the second model to identify cervical tumors. Such features are trained using two lightweight models focused on co-evolutionary neural networks (CNNs). William et al. (41) performed to reduce the probability of mistake by automating the diagnostic process for cervical cancer from Pap-drug photos. Local adaptive histogram equalization was used for image enhancement.

Brats data (24) is used to localize brain tumors in FLAIR scans of MRI. Bilateral flipping is applied to remove noise, while the Gabor filter bank creates text-ton map images. A superpixel is generated by segmenting out an image superpixel is generated by segmenting-level features include at each superpixel histogram level of texton-map is calculated, and the first-order intensity features are calculated as well. Their major contribution is that they made low-level features significant for the localization of brain tumors at the region level approach by integrating features from texton-map images. The prediction of three classes, namely tumor, non-tumor, and background, was made by giving these extracted features later to the classifier. A crossvalidation technique leave-one-out (LOOCV) for the tumor area localization is applied to outperform the existing solution and achieve a dice score of $88 \%$.

In 2021, Wang et al. (42) worked to solve challenges regarding physical security and over-centralized server problems in wireless medical sensor networks (WMSN). Physically unclonable function (PUF) and cutting-edge blockchain technology are combined to propose a reliable and authentic protocol for WSMN. In addition, to deal with biometric information, fuzzy extractor method has been used. For the evaluation of their proposed method, two schemes are used. Their work outperforms the existing studies by achieving higher accuracy through minimizing computation and communication costs.
Similarly, Xiong et al. (43) provided a design of blockchainbased ECDSA with fault-tolerant batch verification protocol for blockchain-enabled IoMT. In 2021, Khamparia et al. (1) combined a convolutional network with a variational encoder for data classification. The dimensionality of images data can be reduced by using a variational encoder with a softmax layer with the kernel size of $2 \times 2$ and $3 \times 3$. Their architecture outperforms the current ML models. Chen et al. (11) developed Cyto Brain that facilitates in subsequent clinical diagnosis, an artificial intelligence (AI) based system. CytoBrain consists of three main modules: (1) to extract only cell images in a whole slide image efficiently, cervical cell segmentation module has been designed. (2) for the cell classification, vgg 16 is used, and a classifier module is designed; Moreover, the last one is the human-aided diagnosis module which can automatically diagnose cervical cancer based on the classification results of cells on a whole slide image.

\section{DATASET AND PRELIMINARIES}

The dataset ${ }^{1}$ used has been released in The University of California's database at Irvine (UCI). The collection contained existing patient history, practices, and procedures and demographic statistics for 858 instances with 32 features per scenario. The dataset contains several missed features since others are incomplete instances, which were chosen not to tackle any privacy concerns, as privacy and security are the common issues in healthcare record frameworks (44-48). Table 1 displays the dataset characteristics and the missed value for each function.

\section{PROPOSED METHODS}

Figure 1 illustrates the flow of our work in a block diagram consisting of pre-processing and classification of cervical cancer. The proposed approach consists of pre-processing and classification of cancer using ML algorithms and deep learning algorithms.

Algorithm 1 shows the working of CervDetect. Random samples have been collected from the input cervical cancer risk factor dataset. For each collected sample, a decision tree has been made to make a prediction. Counting has been done for each final score, and the features are ranked according to their importance. Selected features are input to the shallow neural network where $x_{i}(\mathrm{i}=1,2, \ldots \mathrm{n})$ are the number of input features, $w_{21}$ and $w_{22}$ are the weights and $\mathrm{B} 1$ is constant. $\mathrm{y}$ stores the features convolved with a randomly initialized filter matrix. $\mathrm{V}$ store results of applying a linear transformation on y. $O_{1}$ stores the output generates after applying a sigmoid function on $y$, and e calculates the change in error concerning weights. Infeed backward change error to the weights has been calculated with the help of the chain rule, and the weights are updated.

\subsection{Data Pre-processing}

The dataset regarding cervical cancer has many missed values. A missing value can imply a variety of differences. Records with

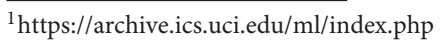


TABLE 1 | Dataset description.

\begin{tabular}{|c|c|c|}
\hline Serial number & Features names in dataset & $\begin{array}{l}\text { No of missing } \\
\text { values }\end{array}$ \\
\hline 1 & "Number of sexual partners" & 26 \\
\hline 2 & "First sexual intercourse" & 7 \\
\hline 3 & "Num of pregnancies" & 56 \\
\hline 4 & "Smokes" & 13 \\
\hline 5 & "Smokes (years)" & 13 \\
\hline 6 & "Smokes (packs/year)" & 13 \\
\hline 7 & "Hormonal Contraceptives" & 108 \\
\hline 10 & "Hormonal Contraceptives (years)" & 108 \\
\hline 11 & "IUD" & 117 \\
\hline 12 & "IUD (years)" & 117 \\
\hline 13 & "STDs" & 105 \\
\hline 14 & "STDs (number)" & 105 \\
\hline 15 & "STDs:condylomatosis" & 105 \\
\hline 16 & "STDs:cervical condylomatosis" & 105 \\
\hline 17 & "STDs:vaginal condylomatosis" & 105 \\
\hline 18 & "STDs:syphilis" & 105 \\
\hline 20 & "STDs:pelvic inflammatory disease" & 105 \\
\hline 21 & "STDs:genital herpes" & 105 \\
\hline 22 & "STDs:molluscum contagiosum" & 105 \\
\hline 23 & "STDs:AIDS" & 105 \\
\hline 24 & "STDs:HIV" & 105 \\
\hline 25 & "STDs:Hepatitis B" & 105 \\
\hline 26 & "STDs:HPV" & 105 \\
\hline 27 & "STDs: Time since first diagnosis" & 787 \\
\hline 28 & "STDs: Time since last diagnosis" & 787 \\
\hline 29 & "Age" & 0 \\
\hline 30 & "STDs: Number of diagnosis" & 0 \\
\hline 31 & "Dx:Cancer" & 0 \\
\hline 32 & "Dx:CIN" & 0 \\
\hline 33 & “Dx:HPV" & 0 \\
\hline 34 & "Dx" & 0 \\
\hline 35 & "Hinselmann" & 0 \\
\hline 36 & "Schiller" & 0 \\
\hline
\end{tabular}

missed values could be included, omitted, or the mean of the variable can be replaced for missing numerical characteristics or with the most frequent value in the case of categorical features. The technique of eliminating the amount of data with missing rows of values decreased from 858 to 737 when applied. We aim to scale the number of features but not the number of input vectors used in the dataset. The following steps are included to pre-process data and make it suitable input for the classifier.

1. Nan: To see relations between variables to treat Nan. Nevertheless, according to the data, the scale of more than 100 Nan values may impact results. We fill features with a median value of less than 100 Nan values in them with median values.

2. Hormonal contraception: There are so many Nan values during the data diagnosis information. Because of this, we cannot calculate the impact of this information, and must
Algorithm 1 CervDetect Algorithm

Input: Cervical cancer risk factor dataset.

1: Begin with the collection of random samples from a dataset.

2: First, this algorithm must generate a decision tree for each sample.

3: The prediction will come from the decision tree.

4: Counting will be carried out in this stage for each final score.

5: Ultimately, pick the most elected outcome of the prediction as to the outcome of the prediction.

6: Output: Rank features with according to their importance.

7: Input: selected features input to shallow neural network $x_{i}(\mathrm{i}=1,2,3,4 \ldots \mathrm{n})$

8: Initialization is the first step after the configuration of the neural network

9: Initiate all weights $w_{21}, w_{22}$ with a random number from the usual distribution, i.e. $\mathrm{N}(0,1)$.

10: Put all of the bias nodes $\mathrm{B} 1=\mathrm{B} 2=1.0$.

11: Feedforward

12: for $I \in l$ do

13: each of $\mathrm{x}$ input have weights $\mathrm{w} 21, \mathrm{w} 22 \ldots$

14: $\mathrm{y}=w_{21} x_{1}, w_{2} 2 x_{2}, \ldots, w_{m} x_{i}$,

15: $\quad \mathrm{v}=\operatorname{sigmoid}(\mathrm{y})$

16: $o_{1}=y_{v 1} w_{21}$

17: $\quad o_{v}=o_{1}$

18: $\quad \mathrm{e}=\frac{1}{n} \sum_{i=1}^{2}\left(y_{i} \text {-ovi }\right)^{2}$

19: end for

20: Feed backward

1: for $i \in k<l$ do

22: Partial derivative of the e to the weight adjusted $w_{i}^{k+1}$ $w_{i k}-\Delta /\left(\varphi w_{k^{i}}\right)$

23: $\quad$ weight update $e_{i}=e_{i} \Delta+w_{i}^{k+1}$

4: end for

eliminate them. Instead, using the Pearson correlation, we will decide which attribute influences hormonal contraceptives. We fill Nan values with correlated attributes according to the resultant heat map. If the patient is older than the mean sample or the amount of pregnancy is less than the mean, the patient may take hormonal contraceptives. Nan values in HC (years) fill with median values using $\mathrm{HC}$ attributes.

3. IUD: Using the Pearson correlation, we can decide which attribute is the "IUD" influencer. Age and amount of maternity factors have an impact on the IUD feature. This indicates that $80 \%$ of patients taking IUD are older than the average age. $70 \%$ of patients who do not take IUD have fewer births than the mean amount of pregnancies. We can fill in the remaining Nan IUD feature values. With the IUD (years) rule, we can fill the -1 values with the IUD rule. If the patient takes IUD, then the UID (years) will be non-zero, so we need to adjust it to mean values.

4. STDs: condylomatosis and STDs: vulvo-perineal condylomatosis has the "STD" effect. We never consider "STD (number)" and "STDs: number of diagnoses" as they are the same attributes as "STDs." Based on our analysis of STDs, 


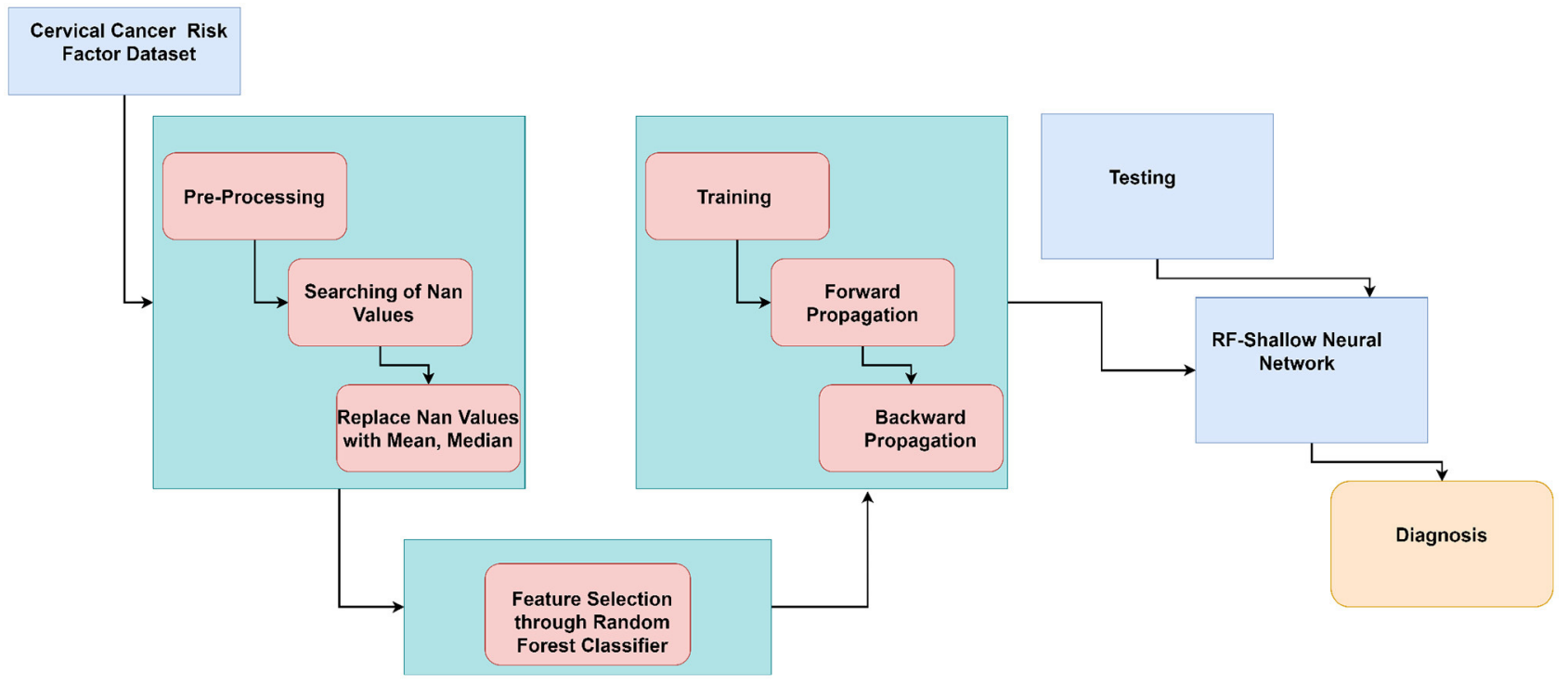

FIGURE 1 | Block diagram of proposed work flow.

we can conveniently fill the Nan values of 1 or zero, and if the individual has either of the STDs, then, the STDs should be 1, the other one will be 0 .

5. STD diseases: According to information, $73 \%$ of non-smoking patients have STD. We do not have Nan values $75 \%$ of patients who do not take IUD are even STDs. Even STDs (number) are the same attribute as STDs. The median case is not helpful because we use mean values for replacement. As per the heat map and our domain awareness, all STD diseases rely on the STDs function and STD (number). We fill Nan values using median for specific values since all STDs rely on certain STDs, and we cannot be assured of a person's disease.

6. AIDS: The correlation function does not give us any hints. However, we recognize that AIDS is also a condition of STDs. So, we fill in the Nan values.

7. STDs-Hepatitis B: This aspect is the result of the STDs-HIV factor. Here we have one patient with a disease, and this importance is negligible compared to the population. The value of Nan is filled with 0 .

8. STDs-HPV: The positive values of HPV are not enough. Zero values cannot be determined by using significant attributes, and we filled the value of Nan with 0 .

9. STDs-time after the first diagnosis and STDs-time after the last diagnosis: When the patient's STDs are negative, the first and last diagnoses cannot be positive. This assumption fills the Nan values in these attributes. Figure 2 illustrates the Pearson correlation between attributes in the cervical cancer risk factor dataset after handling missing values.

\subsection{Random Forest}

The RF model utilizes a sequence of decision trees, where each tree in the forest has been trained using a bootstrap sample of data items, so each tree splitting attribute is picked from a random subset of features. The categorization of patterns shall be based on the collective vote of all trees in the forest. Attribute importance is measured as the decreases in the node's impurity, weighted by the likelihood of approaching the node. The likelihood of the node can be determined by the number of instances that enter the node, separated by the total number of instances. The greater the value, the more important the characteristic is. In Scikit-learn Decision tree, approximates the value of the nodes using Gini significance, considering just two child nodes (binary tree):

$$
\text { nodei }_{j=}=\text { Weight }_{j} C_{j}-W_{l(j)} C_{l(j)}-W_{r(j)} C_{r(j)}
$$

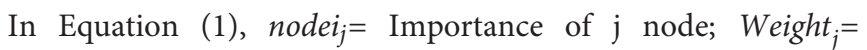
Weighted samples that reach at node $\mathrm{j} ; C_{j}=$ Impurity of $\mathrm{jth}$ node; $l(j)=$ From left split child node on $j$ th node; $r(j)=$ From right split child node on jth node. Each feature importance calculated at decision tree is given by Equation (2):

$$
\text { featureimportance } i_{i}=\frac{\sum j n_{i(j)}}{\sum k n_{i k}}
$$

Where, $\sum \mathrm{j}=$ splitting of a feature I at node $\mathrm{j} ; n_{i, j}=$ node $\mathrm{j}$ importance; $\sum \mathrm{k}=$ all nodes. In Equation (3), normalizing these values between 0 and 1 is done by taking the sum of all feature's importance values and then dividing them.

$$
\text { normalized } f_{i}=\frac{f_{i(\text { feature importance })}}{\sum j f_{j}(\text { all features })}
$$

In Equation (4) at the RF stage, the final feature of importance is the sum, including all trees. Estimating an attribute's significance at each tree is calculated and divided by the overall tree species.

$$
\text { Random forest } i_{i} \frac{\sum j \text { normalized } i_{i j}}{T}
$$




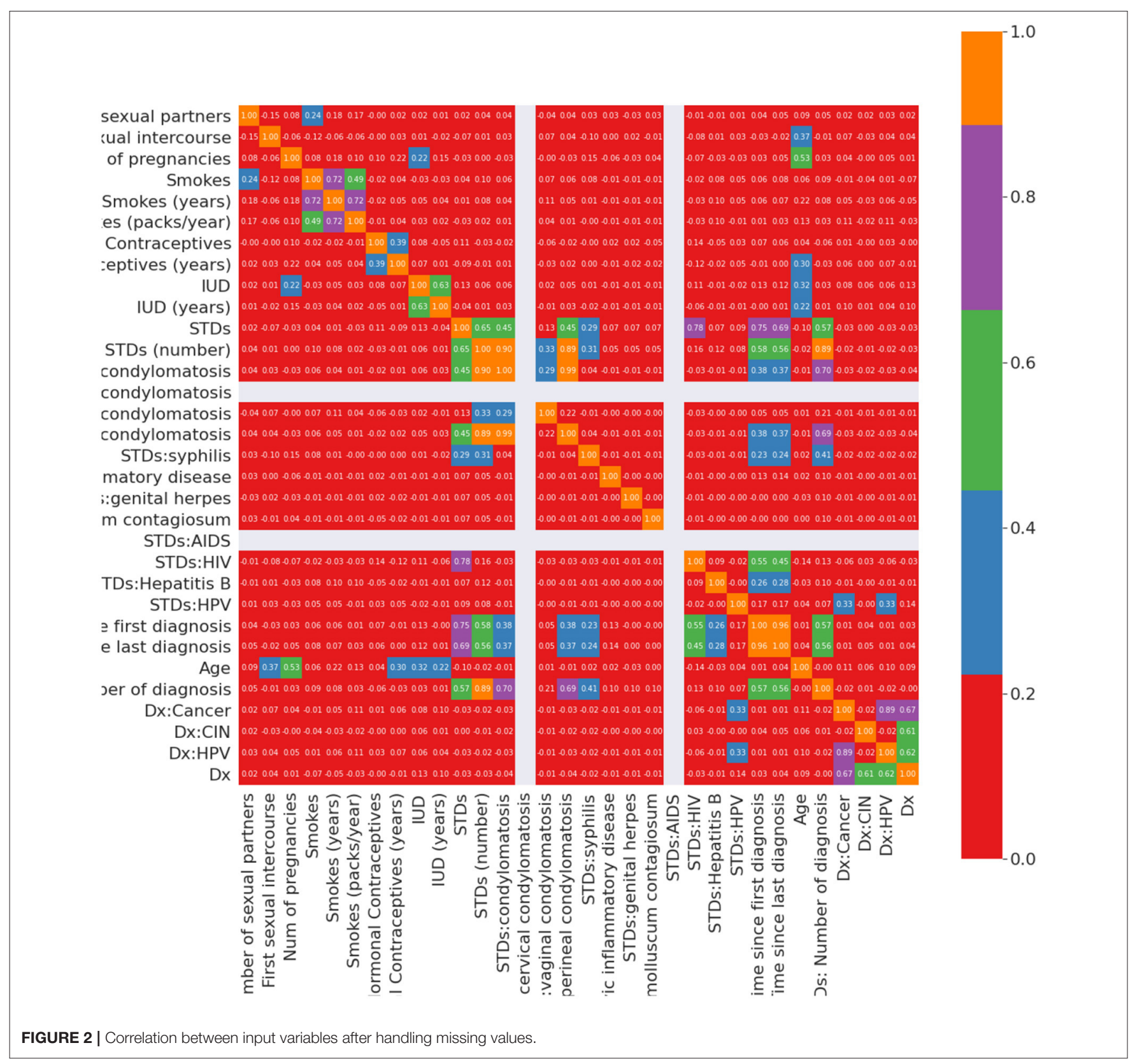

Normalized $f_{i, j}$ is the normalized feature importance for I feature at $j$ th tree, and $\mathrm{T}$ is the total number of trees in the forest. Figure 3 illustrates the feature importance graph that improves the quality of the training dataset.

\subsection{Artificial Neural Networks}

Artificial neural network models are influenced by the human brain structure, which interconnects many biological neurons that are important for maintaining coherent communication. The ANN architecture is computer-based and consists of a variety of simple parallel processing units (49). It is a common statistical technique that can analyze exact relationships between variables. There are several parallel layers in the ANN models, and each layer consists of several neurons. Input, hidden, and output layers are three different types of layers. No calculations are conducted on the input layer. Only feature variables are input. The hidden layer is the key part of the ANN and includes different neurons commonly detected by checking and error. The linear and non-linear functions for the hidden layer neurons are required. The first stage is where the hidden layer neurons receive the input variables compounded by the corresponding correlations (weights), and the second cycle is followed by a nonlinear induction system, usually a sigmoid. A fully connected ANN consists of neurons. The neurons are split into layers composed of one input layer, one output layer, and numerous hidden layers with the contribution of each layer in the next 


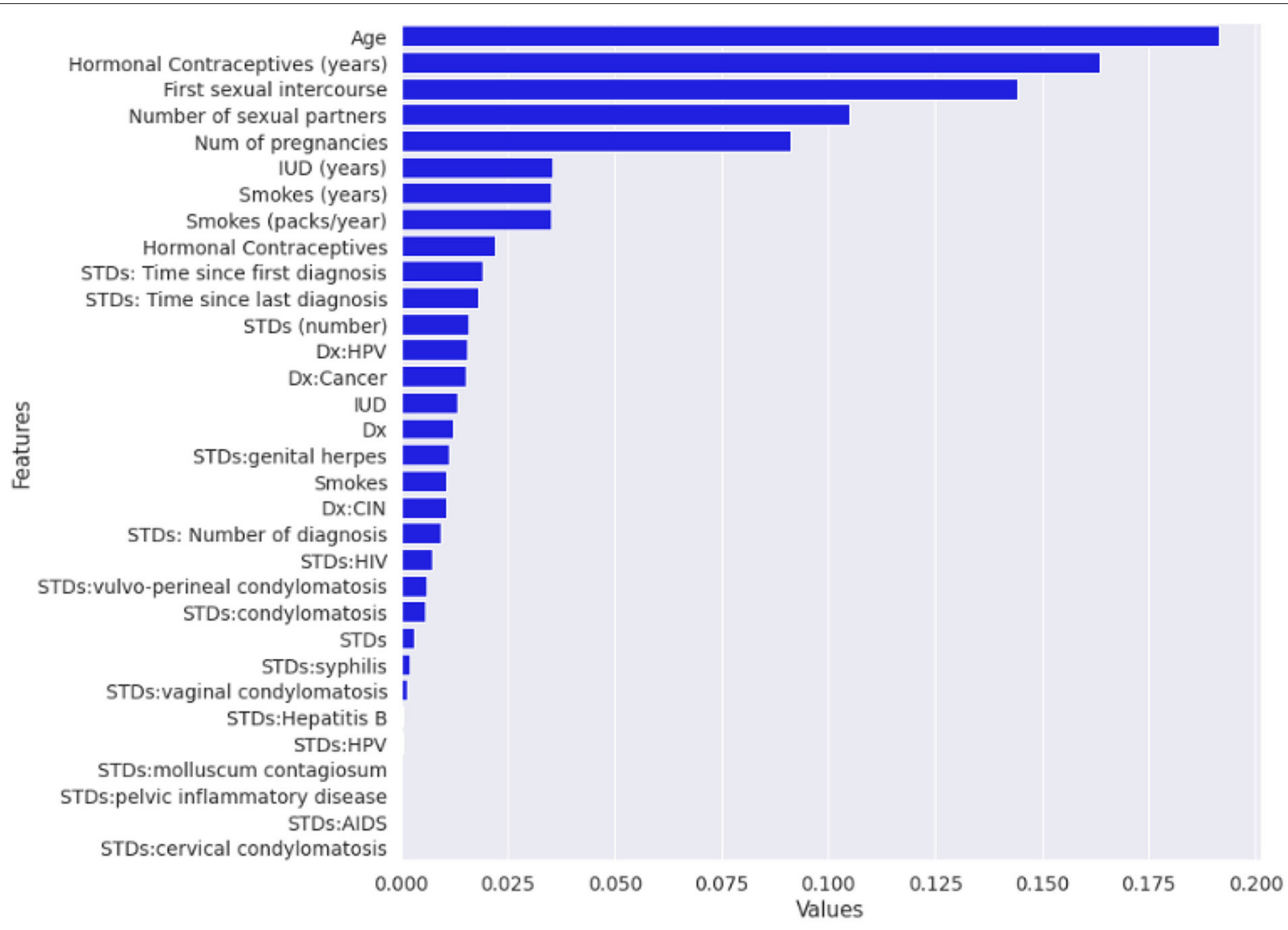

FIGURE 3 | Graph plotted after applying random forest (RF) algorithm for feature importance.

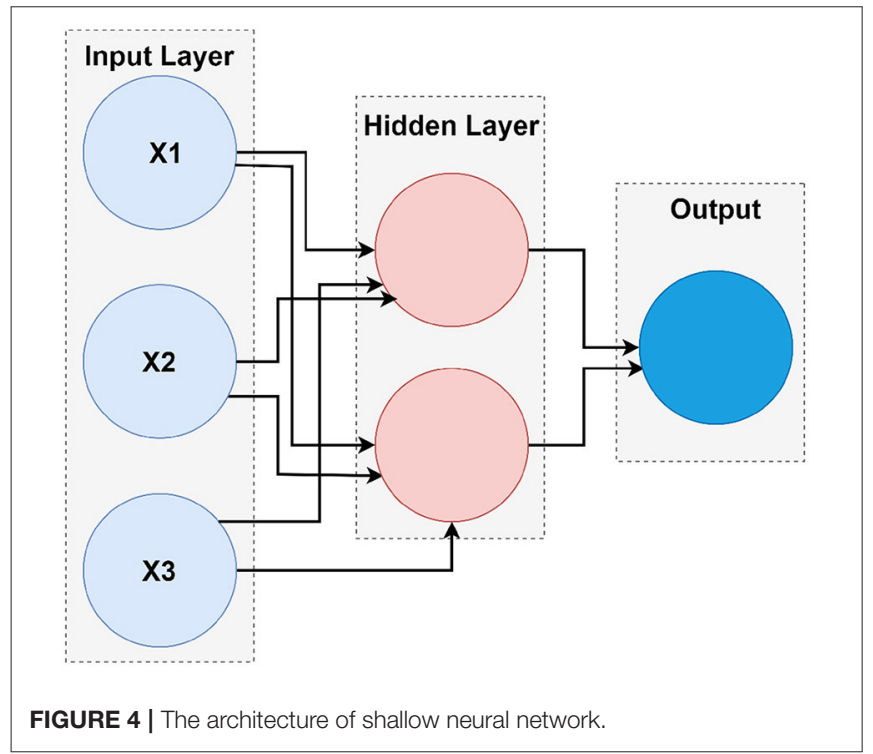

layer as an input in our research, and we concentrate on neural networks with only one layer of hidden data, hidden neurons, and a single output.

\subsection{Shallow Neural Network Architecture}

Different types of neural networks, both shallow and deep, have been built. The terms "shallow" and "deep" refer to the neural network with a minimum number of layers typically known to have a common hidden layer. Deep neural nets are used by neural networks that contain several deep layers. These types of networks execute various tasks, and the fundamental framework of shallow networks enables them to do so. Almost all shallow networks had an input layer, a single hidden layer, and an output layer. The number of hidden nodes in the layer is the only different hyper-parameter. Networks use online learning instead of batch learning, which uses simple backpropagation and gradient descent. Besides, a scaled conjugate gradient descent (SCG) backpropagation algorithm was used in shallow networks. Figure 4 is the fundamental model for shallow neural networks.

The neurons are the atomic component of the neural network. For input data, the output is defined as well as the output is passed to the next layer as an input vector. A neuron may be thought of as a fusion of different segments:

1. The first section calculates the output $Y$, using input parameters and weights.

2. The second section executes the activation on $\mathrm{Y}$ to give the final performance of neuron $\mathrm{A}$. 
The hidden layer consists of several neurons, which each executes below mentioned 5 and 6 equations. Those two neurons found in the hidden layer of the external neural network are evaluated as follows:

$$
\begin{gathered}
Y_{1}^{[1]}=w_{1}^{[1]} T z+b_{1}^{[1]}, a_{1}^{[1]}=\sigma\left(y_{1}^{[1]}\right) \\
Y_{2}^{[1]}=w_{2}^{[1]} T z+b_{2}^{[1]}, a_{2}^{[1]}=\sigma\left(y_{2}^{[1]}\right)
\end{gathered}
$$

Where the superscript number [i] is the number of the layer, and the subscription number $\mathrm{j}$ is the number of the neurons in a particular layer. $\mathrm{Y}$ is an input vector made up of three components.

$W_{i}$ represents the weights connected to each input variable, and bi represents the bias factor connected to each line, hidden line, and output layer. $\mathrm{Z}[\mathrm{i}] \mathrm{j}$ is just the intermediate output associated with the $j$ neurons found in the ith layer. $A[i] j$ is the final output associated with the neuron $\mathrm{j}$ in the ith layer.

\begin{tabular}{lll}
\hline TABLE 2 | Confusion matrix of CervDetect. & & \\
\hline Predicted class /Actual class & Biopsy & Normal \\
\hline Biopsy & TP & FN \\
Normal & FP & TN
\end{tabular}

TABLE 3 | Selected optimal features.

\begin{tabular}{ll}
\hline Serial no & Important features according to their rank \\
\hline 1 & "Age" \\
2 & "Hormonal Contraceptives (years)" \\
3 & "First sexual intercourse" \\
4 & "Num of pregnancies" \\
5 & "IUD (years)" \\
6 & "Smokes (years)" \\
7 & "Smokes (packs/year)" \\
8 & "Hormonal Contraceptives" \\
9 & "STDs: Time since first diagnosis" \\
10 & "STDs: Time since last diagnosis"
\end{tabular}

Sigma is a function of activation that squashes the input value into the range of $(0,1)$. Mathematically, this is described as in Equation (7):

$$
\sigma=\frac{1}{1+e^{-y i}}
$$

Equation (8) represents all $\mathrm{Z}$ intermediate outputs in a single multiplication matrix.

$$
\begin{gathered}
Z^{1}=X^{[1] T} X+b^{1} \\
A_{1}^{[1]}=\sigma\left(Z^{1}\right)
\end{gathered}
$$

The above Equation (9) represents all activation $\mathrm{A}$ in a single multiplication matrix.

To calculate the output for an input vector $y$, the following steps are performed as mentioned in below (10), (11), (12), and (13) equations. These steps can also be called feedforward propagation.

$$
\begin{gathered}
Z^{1}=W^{[1] T} Y+b^{1} \\
A_{1}^{[1]}=\sigma\left(Z^{1}\right) \\
Z^{2}=W^{[2] T} A^{1}+b^{2} \\
Z^{\text {final }}=A^{[2]}=\sigma\left(Z^{2}\right)
\end{gathered}
$$

Where the Equation (10) measures the $\mathrm{Z}$ (18) intermediate output of the first hidden layer. The Equation (11) is used to measure the final production A (18) of the first hidden layer. The Equation (12) calculates the $\mathrm{Z}$ intermediate value of a processing layer Z. The Equation (13) calculates the end product A of the

TABLE 4 | Accuracy table.

\begin{tabular}{llc}
\hline serial no & Performance measures & Performance $\%$ \\
\hline 1 & Accuracy & $93.6 \%$ \\
2 & TPR & $100 \%$ \\
3 & FPR & $100 \%$
\end{tabular}

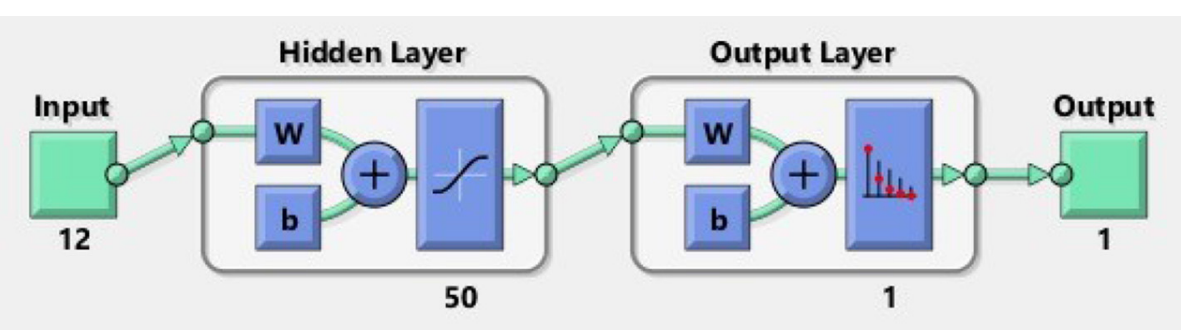

FIGURE 5 | Shallow neural network. 
output layer, which is now the result of the whole neural network. When the results are obtained from each neuron's hidden layer, they are transferred to the next layer, where each neuron in the output layer finalizes the values.

$$
\begin{gathered}
Y_{1}^{[2]}=w_{1}^{[2]} \text { out }\left(y_{1}\right)+w_{2}^{[2]} \text { out }\left(y_{2}\right)+. .+w_{14}^{[2]} \text { out }\left(y_{14}\right)+b_{2}^{[2]} \\
a_{1}^{[2]}=\sigma\left(Y_{1}^{[2]}\right) \\
Y_{15}^{[2]}=w_{30}^{[2]} \text { out }\left(y_{1}\right)+w_{31}^{[2]} \text { out }\left(y_{2}\right)+. .+w_{43}^{[2]} \operatorname{out}\left(y_{15}\right)+b_{2}^{[2]} \\
a_{50}^{[2]}=\sigma\left(Y_{50}^{[2]}\right)
\end{gathered}
$$

Equations (14- 17) are used to determined the error after the results are obtained for each neuron in the output layer. When this is the critical error, it will avoid back-propagating to change the previous weights to get the minimal error in the feedforward process.

\section{RESULTS AND DISCUSSION}

In our work, the most significant performance measure for cervical cancer diagnosis accuracy is used to calculate the RF-shallow neural network's performance. The true positive (TP) is identical to those rejected, representing the number of cancer patients marked as Biopsy. False-positive (FP) is the inverse to deny wrongly and represents normal patients as cancer patients. The true negative (TN) is equal to those correctly identified, representing the number of normal patients identified as normal. False-negative (FN) is equal to any incorrectly identified, representing the number of cancer patients identified as normal patients. Table 2 shows the confusion matrix.

Accuracy: the proportion of the number of patient records correctly categorized against the overall number of patient records in the dataset as shown in Equation (18).

$$
A C=\frac{T P+T N}{T p+T N+F P+F N}
$$

True positive ratio (TPR): it is identical to detection rate (DR). TPR indicates the proportion of the number of patient records
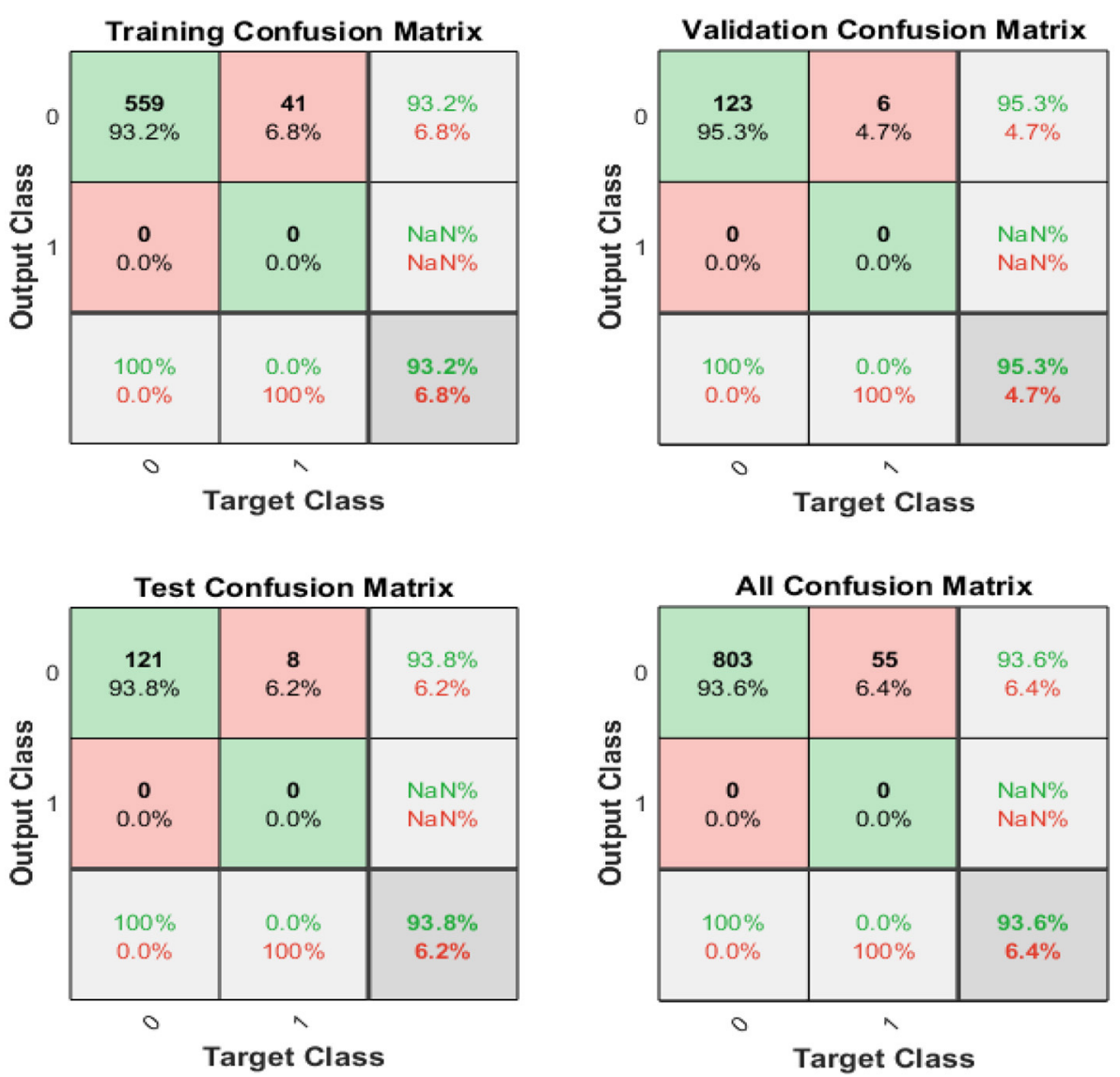

FIGURE 6 | Confusion matrix of training, testing, and validation. 

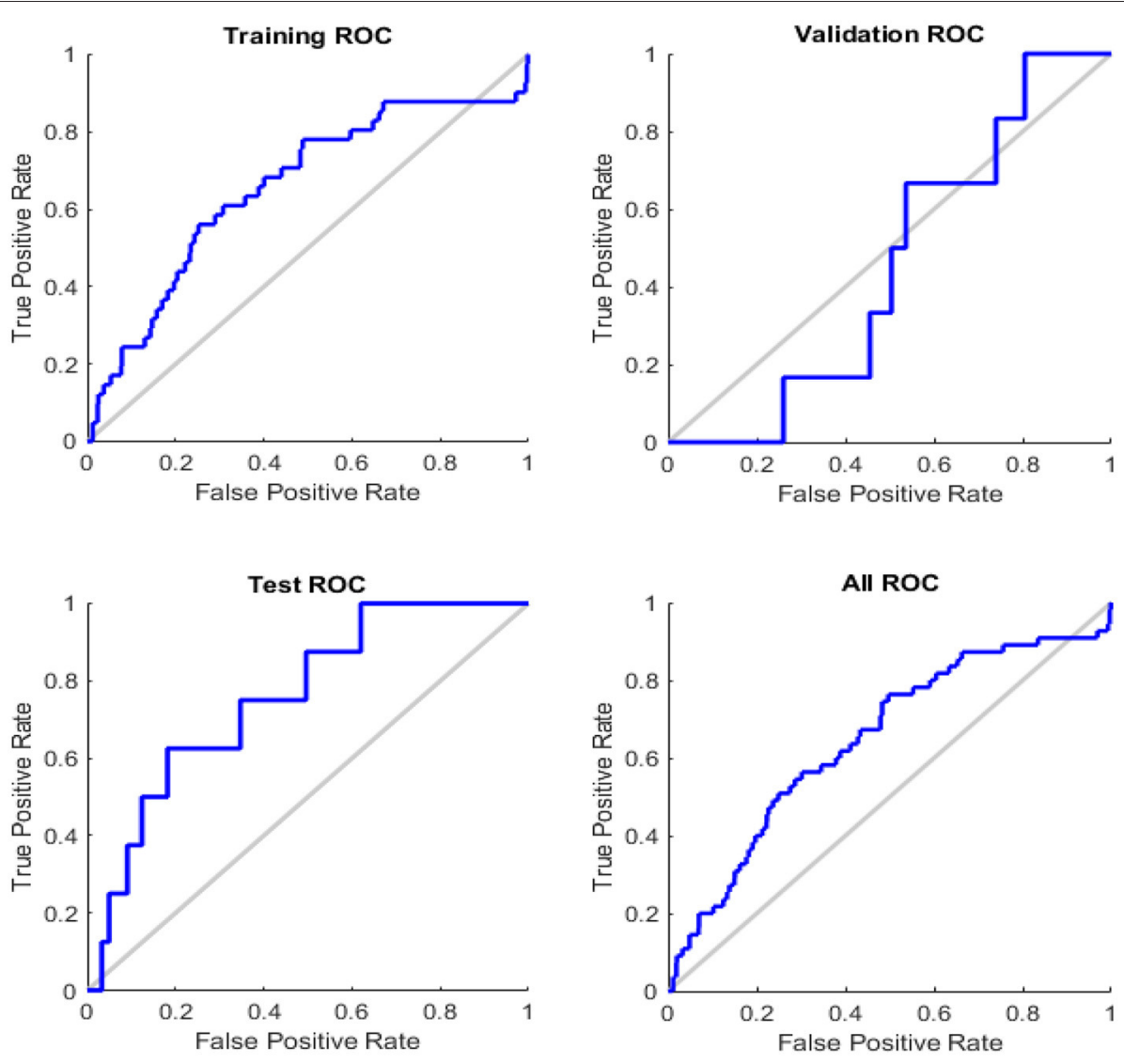

FIGURE 7 | Roc curve of testing, training, and validation.
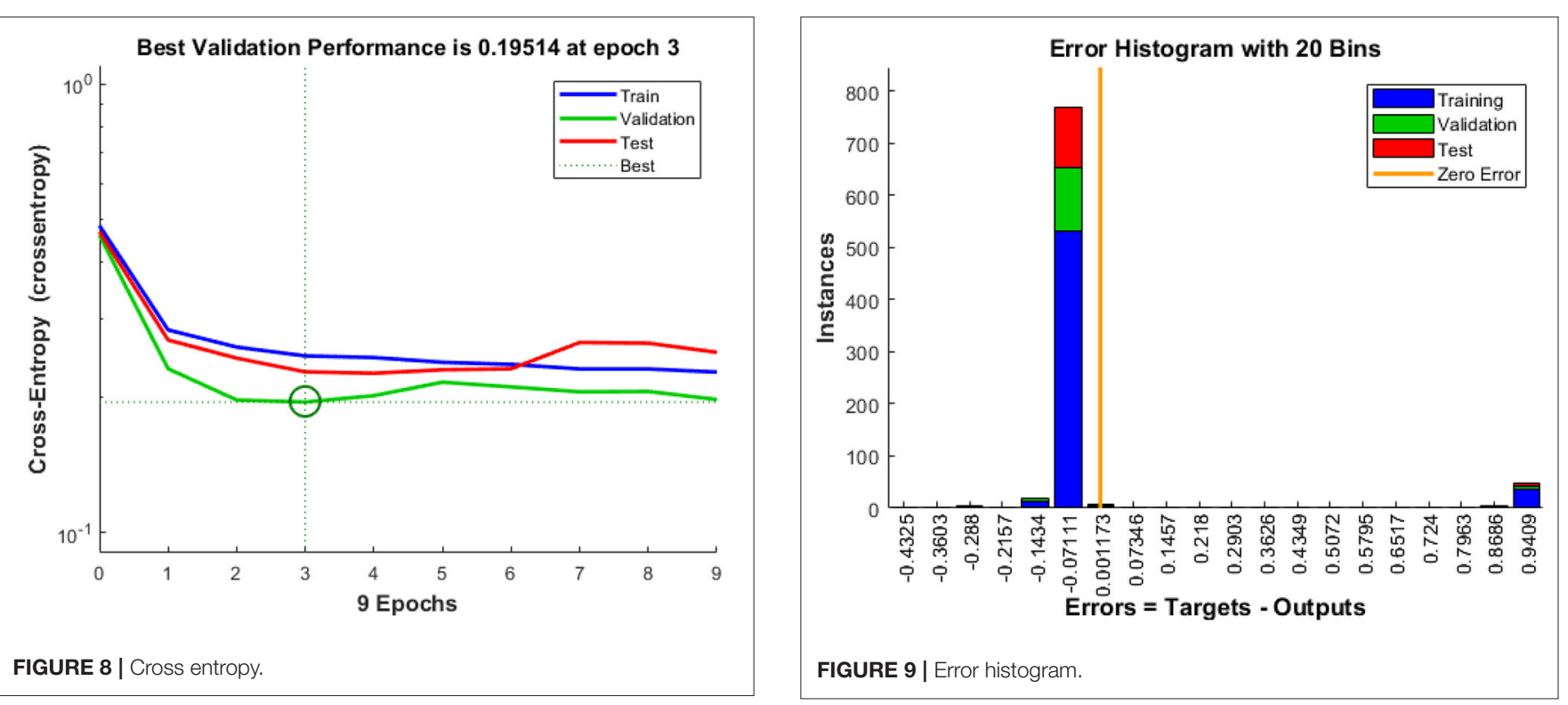


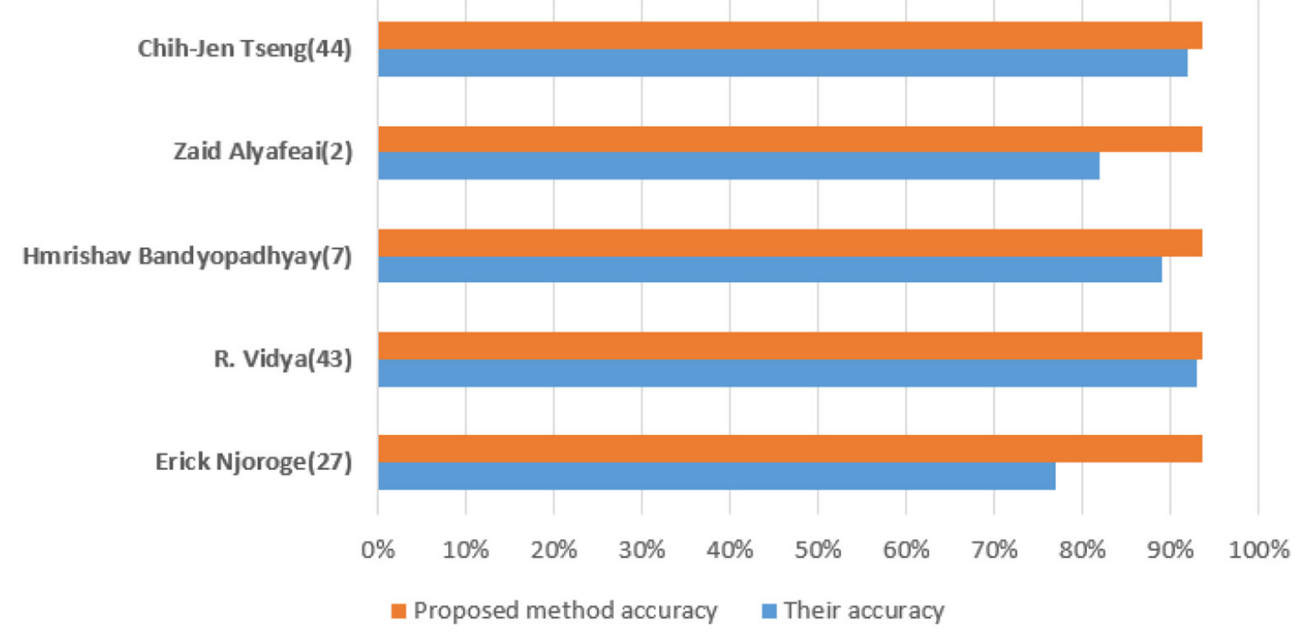

FIGURE 10 | Comparative analysis with existing works.

correctly identified over the overall patient records, as shown in Equation (19).

$$
T P R=\frac{T P}{T P+F N}
$$

False positive ratio (FPR): the ratio of the numbers of incorrectly declined records divided by the cumulative amount of total record as shown in Equation (20)

$$
F P R=\frac{F P}{F P+T N}
$$

In this study, we have proposed a new method called RF-shallow neural network to diagnose cervical cancer as the effect of the wrong diagnosis in cervical cancer or vice versa is high. Data mining provides tools and techniques to derive important data from a huge dataset by analysis. Throughout this study, ML models (RF and shallow neural network) were used for cervical cancer diagnostics to demonstrate the importance of building the model with data cleaning, the replacement of null values, and the implementation of a feature selection procedure to achieve a better accuracy prediction for an optimal function subset. This methodology used ML methodologies for cervical cancer data to assess the efficacy of classifier models by considering all medical records. Dataset missing records are filled by replacing missing values in rows with their mean and median by finding the Pearson correlation between variables. The RF's implementation as a feature selection technique and the shallow neural network training by an optimum subset of features was chosen based on the variables' value. In addition to the one target attribute biopsy for cervical cancer diagnosis, the following attributes in Table 3 have been defined as more important features among the cervical cancer risk factors dataset.

A shallow neural network consists of one input layer, one layer of hidden neurons, and a single output layer, as shown in Figure 5. Neural pattern recognition uses the scaled conjugate gradient algorithm. The value of one class, "0," is treated as a negative test for a cervical cancer patient, and the value of another class, "1," is considered a positive measure for a cervical cancer patient and recommended for biopsy.

A total of 12 optimum features input space has been created after their importance and ranking. We took $70 \%$ of the data from 859 records for training, $15 \%$ for validation, and $15 \%$ for testing. To obtain the results, we used 50 hidden neurons and 1 epoch. Table 4 shows the findings we obtained from ANN using scaled conjugate gradient using minimized cross-entropy, correct true positive rate, false-positive rate, and accuracy.

Figure 6 displays the confusion matrixes for validation, testing, and training, and the three pieces of information merged. The network findings are promising because we can conclude with many correct responses in green squares and a minimal number of wrong responses in red squares. The lower squares indicate the actual precision.

Figure 7 illustrates the receiver operating feature (ROC) curve. The colored lines in each axis represent the curves of a ROC. The ROC curve is a graphical representation of a true positive (sensitivity) vs. the false positive (1-specificity) in which the threshold has been changed. The optimal search has shown points in the upper left corner with $93 \%$ accuracy.

There have been several lines, train, validation, testing, and best in this study, as shown in Figure 8. In general, the ideal (dotted) line is that the other will lie on or above this (dotted) line, and we can conclude that the training has been carried out effectively. If all of the 3 (training, validation, and testing) lines cross or travel past the ideal (dotted) path, this implies that convergence has been completed. If this is not the case, retrain the network.

When the model is tested for more than 129 tests, the histogram displayed in Figure 9 shows the error that occurs during the test process. The distribution of the error size of the histogram shows how well the neural network matches the 

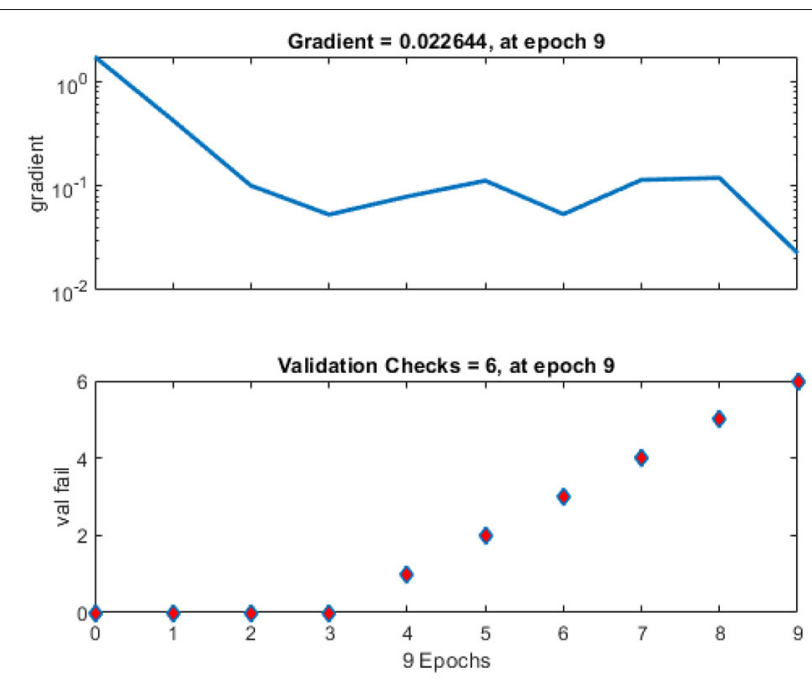

FIGURE 11 | Training state of neural network for cervical cancer diagnosis.

results. It indicates errors that the solution to mean squared error (MSE) in our scenario is good. More than 500 events are utilized for training purposes. Approximately, 129 are used for validation, and the remaining 129 is used to check which indicates an error of 0.07111 .

Figure 10 shows a training state that represents the actual progress/state of the training at a given period when the training is in progress. In our situation, 6 validation errors are stated, i.e., unless 6 validation tests are performed, then training will end. Validation test error implies that the dataset has specific issues; a training algorithm cannot recognize certain instances. This indicates that due to dataset issues, validation test errors may be produced. In our case, 6 validity tests are included. As shown in Figure 11, modified CervDetect achieved 93.6\% that outperforms the existing work.

\section{CONCLUSION AND FUTURE WORK}

This study provides a risk factor for cervical cancer. The cure percentage, the number of female cases recovered from cancer may be improved by recognizing risk factors for cervical cancer. Data mining techniques in the medical industry have an immense capacity to develop diagnostic and prognosis indicator applications that can aid in the proper initial treatment of

\section{REFERENCES}

1. Khamparia A, Gupta D, Rodrigues JJ, de Albuquerque VHC. DCAVN: cervical cancer prediction and classification using deep convolutional and variational autoencoder network. Multimedia Tools Appl. (2021) 80:30399415. doi: 10.1007/s11042-020-09607-w

2. Abbas S, Jalil Z, Javed AR, Batool I, Khan MZ, Noorwali A, et al. BCD-WERT: a novel approach for breast cancer detection using whale optimization based efficient features and extremely randomized tree algorithm. PeerJ Comput. Sci.. (2021) 7:e390. doi: 10.7717/peerj-cs.390 life-threatening diseases. With various data mining techniques, it is important to enhance the standard of care in hospitals and maximize patients' recovery rates. The outcomes of the data reduction also continued to increase the efficiency of the methods. Generally, the less critical features used in the classification process, the stronger the external neural network's efficiency. In this article, we present CervDetect, a hybrid approach that combines RF and shallow neural network to understand the risk elements of malignant cervical formation by deploying machine learning algorithms on medical data. CervDetect utilizes Pearson correlation between input variables and the output variable to pre-process the data. The RF feature selection technique is used to select significant features. CervDetect effectively enhances the detection rate in comparison with state-of-the-art studies. Results show that the proposed approach achieved an accuracy of 93.6\%, MSE error of 0.07111 , FPR of $6.4 \%$, and FNR of $100 \%$. For future work, applying a sequential model to this dataset, such as a recursive deep neural network, can be useful for diagnosis. Due to the severity of the disease, cervical cancer is chosen as a starting point. The same work delineated here could be extended to other gynecological cancer predictions and other disease entities. Thus, the provided approach CervDetect showed optimal performance accuracy with the optimum features dataset.

\section{DATA AVAILABILITY STATEMENT}

The original contributions presented in the study are included in the article/supplementary material, further inquiries can be directed to the corresponding author/s.

\section{AUTHOR CONTRIBUTIONS}

SA and MM: conceptualization. MM: data curation. SA: formal analysis, investigation, methodology, and software. MR: funding acquisition. MG and MR: project administration, resources, and writing-review and editing. MG and SA: supervision. MM and MG: validation and visualization. All authors contributed to the article and approved the submitted version.

\section{ACKNOWLEDGMENTS}

This research has been supported by the Department of Information Systems, Faculty of Management, Comenius University in Bratislava, Slovakia. 
6. Javed AR, Faheem R, Asim M, Baker T, Beg MO. A smartphone sensors-based personalized human activity recognition system for sustainable smart cities. Sustain Cities Soc. (2021) 71:102970. doi: 10.1016/j.scs.2021.102970

7. Aslam B, Javed AR, Chakraborty C, Nebhen J, Raqib S, Rizwan M. Blockchain and ANFIS empowered IoMT application for privacy preserved contact tracing in COVID-19 pandemic. Pers Ubiquit Comput. (2021) 1-17. doi: 10.1007/s00779-021-01596-3

8. Iwendi C, Mahboob K, Khalid Z, Javed AR, Rizwan M, Ghosh U. Classification of COVID-19 individuals using adaptive neuro-fuzzy inference system. Multimedia Syst. (2021) 1-15. doi: 10.1007/s00530-021-00774-w

9. Tripathy B, Parimala M, Reddy GT. Innovative classification, regression model for predicting various diseases. In: Data Analytics in Biomedical Engineering and Healthcare. Elsevier (2021). p. 179-203.

10. Javed AR, Sarwar MU, Khan S, Iwendi C, Mittal M, Kumar N. Analyzing the effectiveness and contribution of each axis of tri-axial accelerometer sensor for accurate activity recognition. Sensors. (2020) 20:2216. doi: 10.3390/ s20082216

11. Chen H, Liu J, Wen QM, Zuo ZQ, Liu JS, Feng J, et al. CytoBrain: cervical cancer screening system based on deep learning technology. J Comput Sci Technol. (2021) 36:347-60. doi: 10.1007/s11390-021-0849-3

12. Javed AR, Sarwar MU, Beg MO, Asim M, Baker T, Tawfik H. A collaborative healthcare framework for shared healthcare plan with ambient intelligence. HHum Centric Comput Inform Sci. (2020) 10:1-21. doi: 10.1186/s13673-020-00245-7

13. Javed AR, Fahad LG, Farhan AA, Abbas S, Srivastava G, Parizi RM, et al. Automated cognitive health assessment in smart homes using machine learning. Sustain Cities Soc. (2021) 65:102572. doi: 10.1016/j.scs.2020.102572

14. Sarwar MU, Javed AR. Collaborative health care plan through crowdsource data using ambient application. In: 2019 22nd International Multitopic Conference (INMIC). IEEE (2019). p. 1-6.

15. Parkin DM, Bray F, Ferlay J, Pisani P. Global cancer statistics, 2002. CA Cancer J Clin. (2005) 55:74-108. doi: 10.3322/canjclin.55.2.74

16. Salmi N, Rustam Z. Naive Bayes classifier models for predicting the colon cancer. In: IOP Conference Series: Materials Science and Engineering. vol. 546. IOP Publishing (2019). p. 052068.

17. Chirenje ZM, Rusakaniko S, Kirumbi L, Ngwalle EW, Makuta-Tlebere P, Kaggwa S, et al. Situation analysis for cervical cancer diagnosis and treatment in east, central and southern African countries. Bull World Health Org. (2001) 79:127-32.

18. Torre LA, Bray F, Siegel RL, Ferlay J, Lortet-Tieulent J, Jemal A. Global cancer statistics, 2012. CA Cancer J Clin. (2015) 65:87-108. doi: 10.3322/caac.21262

19. Sarwar A, Ali M, Suri J, Sharma V. Performance evaluation of machine learning techniques for screening of cervical cancer. In: 2015 2nd International Conference on Computing for Sustainable Global Development (INDIACom). IEEE (2015). p. 880-6.

20. Hajimirzaei B, Navimipour NJ. Intrusion detection for cloud computing using neural networks and artificial bee colony optimization algorithm. ICT Express. (2019) 5:56-9. doi: 10.1016/j.icte.2018.01.014

21. Prokopowicz P, Mikołajewski D, Tyburek K, Mikołajewska E. Computational gait analysis for post-stroke rehabilitation purposes using fuzzy numbers, fractal dimension and neural networks. Bull Pol Acad Sci Techn Sci. (2020) $191-8$.

22. Bojja GR, Ofori M, Liu J, Ambati LS. Early public outlook on the coronavirus disease (COVID-19) A social media study. (2020).

23. de Brevern AG, Meyniel JP, Fairhead C, Neuvéglise C, Malpertuy A. Trends in IT innovation to build a next generation bioinformatics solution to manage and analyse biological big data produced by NGS technologies. BioMed Res Int. (2015) 2015. doi: 10.1155/2015/904541

24. Rehman ZU, Zia MS, Bojja GR, Yaqub M, Jinchao F, Arshid K. Texture based localization of a brain tumor from MR-images by using a machine learning approach. Med Hypotheses. (2020) 141:109705. doi: 10.1016/j.mehy.2020.109705

25. Deepa N, Prabadevi B, Maddikunta PK, Gadekallu TR, Baker T, Khan MA, et al. An AI-based intelligent system for healthcare analysis using Ridge-Adaline Stochastic Gradient Descent Classifier. J Supercomput. (2021) 77:1998-2017. doi: 10.1007/s11227-020-03347-2
26. Lee CKH, Tse YK, Ho G, Chung SH. Uncovering insights from healthcare archives to improve operations: an association analysis for cervical cancer screening. Technol Forecast Soc Change. (2021) 162:120375. doi: $10.1016 /$ j.techfore.2020.120375

27. Punjani DN, Atkotiya KH. Cervical cancer prediction using data mining. Int J for Res Appl Sci Eng Tech. (2017) 5:1856.

28. Breiman L. Random Forests. Statistics Department. Berkeley, CA: University of California (2001).

29. Sun G, Li S, Cao Y, Lang F. Cervical Cancer Diagnosis based on Random Forest. Int J Performabil Eng. (2017) 13:446-57. doi: 10.23940/ijpe.17.04.p12.446457

30. Mittal K, Aggarwal G, Mahajan P. Performance study of K-nearest neighbor classifier and $\mathrm{K}$-means clustering for predicting the diagnostic accuracy. Int $\mathrm{J}$ Inform Technol. (2019) 11:535-40. doi: 10.1007/s41870-018-0233-x

31. Mitra P, Mitra S, Pal SK. Staging of cervical cancer with soft computing. IEEE Trans Biomed Eng. (2000) 47:934-40. doi: 10.1109/10.846688

32. $\mathrm{Wu} \mathrm{W}$, Zhou H. Data-driven diagnosis of cervical cancer with support vector machine-based approaches. IEEE Access. (2017) 5:25189-95. doi: 10.1109/ACCESS.2017.2763984

33. Ashok B, Aruna P. Comparison of Feature selection methods for diagnosis of cervical cancer using SVM classifier. Int J Eng Res Appl. (2016) 6:94-9.

34. Tseng CJ, Lu CJ, Chang CC, Chen GD. Application of machine learning to predict the recurrence-proneness for cervical cancer. Neural Comput Appl. (2014) 24:1311-6. doi: 10.1007/s00521-013-1359-1

35. Reif DM, Motsinger AA, McKinney BA, Crowe JE, Moore JH. Feature selection using a random forests classifier for the integrated analysis of multiple data types. In: 2006 IEEE Symposium on Computational Intelligence and Bioinformatics and Computational Biology. IEEE (2006). p. 1-8.

36. Vidya R, Nasira G. Prediction of cervical cancer using hybrid induction technique: A solution for human hereditary disease patterns. Indian J Sci Technol. (2016) 9:1-10. doi: 10.17485/ijst/2016/v9i30/82085

37. Geeitha S, Thangamani M. Integrating HSICBFO and FWSMOTE algorithmprediction through risk factors in cervical cancer. J Ambient Intell Hum Comput. (2020) 1-13. doi: 10.1007/s12652-020-02194-6

38. Anuraga G, Fernanda J, Pebrianty JW. Random forest prognostic factor in colorectal cancer. J Phys Confer Ser. (2019) 1217:012098. doi: 10.1088/1742-6596/1217/1/012098

39. Bandyopadhyay H, Nasipuri M. Segmentation Of Pap Smear Images For Cervical Cancer Detection. In: 2020 IEEE Calcutta Conference (CALCON). IEEE (2020). p. 30-3.

40. Alyafeai Z, Ghouti L. A fully-automated deep learning pipeline for cervical cancer classification. Expert Syst Appl. (2020) 141:112951. doi: 10.1016/j.eswa.2019.112951

41. William W, Ware A, Basaza-Ejiri AH, Obungoloch J. Cervical cancer classification from Pap-smears using an enhanced fuzzy C-means algorithm. Inform Med Unlocked. (2019) 14:23-33. doi: 10.1016/j.imu.2019.02.001

42. Wang W, Qiu C, Yin Z, Srivastava G, Gadekallu TR, Alsolami F, et al. Blockchain and PUF-based lightweight authentication protocol for wireless medical sensor networks. IEEE Intern Things J. (2021). 2021:5580939. doi: 10.1155/2021/5580939

43. Xiong H, Jin C, Alazab M, Yeh KH, Wang H, Gadekallu TRR, et al. On the design of blockchain-based ECDSA with fault-tolerant batch verication protocol for blockchain-enabled IoMT. IEEE J Biomed Health Inform. (2021). doi: 10.1109/JBHI.2021.3112693

44. Mubashar A, Asghar K, Javed AR, Rizwan M, Srivastava G, Gadekallu TR, et al. Storage and proximity management for centralized personal health records using an IPFS-based optimization algorithm. J Circuits Syst Comput. (2021) 2250010. doi: 10.1142/S0218126622500104

45. Kutia S, Chauhdary SH, Iwendi C, Liu L, Yong W, Bashir AK. SocioTechnological factors affecting user's adoption of eHealth functionalities: A case study of China and Ukraine eHealth systems. IEEE Access. (2019) 7:90777-88. doi: 10.1109/ACCESS.2019.2924584

46. Mohiyuddin A, Javed AR, Chakraborty C, Rizwan M, Shabbir M, Nebhen J. Secure cloud storage for medical IoT data using adaptive neuro-fuzzy inference system. Int J Fuzzy Syst. (2021) 1-13. doi: 10.1007/s40815-021-01104-y 
47. Javed AR, Sarwar MU, ur Rehman S, Khan HU, Al-Otaibi YD, Alnumay WS. Pp-spa: privacy preserved smartphone-based personal assistant to improve routine life functioning of cognitive impaired individuals. Neural Process Lett. (2021) 1-18. doi: 10.1007/s11063-020-10414-5

48. Shabbir M, Shabbir A, Iwendi C, Javed AR, Rizwan M, Herencsar N, et al. Enhancing security of health information using modular encryption standard in mobile cloud computing. IEEE Access. (2021) 9:8820-34. doi: 10.1109/ACCESS.2021.3049564

49. Susanty M, Sahrul, Rahman AF, Normansyah MD, Irawan A. Offensive Language Detection using Artificial Neural Network. In: 2019 International Conference of Artificial Intelligence and Information Technology (ICAIIT). IEEE (2019) p. 350-3.

Conflict of Interest: The authors declare that the research was conducted in the absence of any commercial or financial relationships that could be construed as a potential conflict of interest.
Publisher's Note: All claims expressed in this article are solely those of the authors and do not necessarily represent those of their affiliated organizations, or those of the publisher, the editors and the reviewers. Any product that may be evaluated in this article, or claim that may be made by its manufacturer, is not guaranteed or endorsed by the publisher.

Copyright (๑) 2021 Mehmood, Rizwan, Gregus ml and Abbas. This is an open-access article distributed under the terms of the Creative Commons Attribution License (CC $B Y)$. The use, distribution or reproduction in other forums is permitted, provided the original author(s) and the copyright owner(s) are credited and that the original publication in this journal is cited, in accordance with accepted academic practice. No use, distribution or reproduction is permitted which does not comply with these terms. 